\title{
Construção do conceito de promoção da saúde: comparação entre estudantes ingressantes e concluintes de Fonoaudiologia
}

\author{
Construction of the health promotion concept: comparative \\ study between freshmen and senior Speech-Language \\ Pathology and Audiology students
}

\author{
Lidiane Gonçalves dos Santos ${ }^{1}$, Stela Maris Aguiar Lemos ${ }^{2}$
}

\begin{abstract}
RESUMO
Objetivo: Caracterizar e comparar o conhecimento de ingressantes (estudantes do $1^{\circ}$ período) e concluintes (estudantes do $8^{\circ}$ período) de um curso de Fonoaudiologia acerca do tema promoção da saúde. Métodos: Estudo descritivo transversal realizado por meio da aplicação de 92 formulários em estudantes de Fonoaudiologia ingressantes e concluintes. A análise dos dados obtidos foi realizada em três etapas: análise descritiva das questões fechadas, análise estatística da associação entre as respostas do $1^{\circ} \mathrm{e}$ do $8^{\circ}$ períodos, e análise qualitativa das questões abertas do formulário. Resultados: A maioria dos entrevistados cursou disciplinas com o tema promoção da saúde; mais de um terço participou de atividades extracurriculares ou disciplinas práticas envolvendo o tema. A maioria referiu saber o que é promoção da saúde e ter a intenção de realizar ações em promoção da saúde. Os ingressantes relataram conceitos empíricos sobre promoção da saúde e confundiram promoção com prevenção. Entre os concluintes, a confusão existe em menor quantidade. Conclusão: O curso de graduação em Fonoaudiologia dos estudantes entrevistados contribui para a formação do conceito de promoção da saúde, porém existe uma confusão entre os termos prevenção e promoção da saúde, tanto entre os estudantes do $1^{\circ}$ quanto do $8^{\circ}$ período. Os dados evidenciam a importância de discutir o tema promoção da saúde durante o processo de formação do fonoaudiólogo.
\end{abstract}

Descritores: Fonoaudiologia/educação; Promoção da saúde; Conhecimentos, atitudes e prática em saúde; Educação em saúde; Educação superior; Questionários

\section{INTRODUÇÃO}

A promoção da saúde foi um tema amplamente discutido nas conferências internacionais de saúde, como as realizadas em Ottawa (1986), Adelaide (1988), Sundsvall (1991), Santafé (1992) e Jacarta $(1997)^{(1)}$. Diante da complexidade do processo saúde-doença e das condições de saúde das populações de diversos países, percebeu-se que a abordagem assistencialista não seria capaz de solucionar as questões contemporâneas de saúde, como a violência e as doenças não transmissíveis. As estratégias de promoção de saúde, ao proporcionarem o envolvimento de outros setores, tais como o econômico e a educação, além do sanitário, no processo de planejamento e

Trabalho realizado no Curso de Fonoaudiologia, Universidade Federal de Minas Gerais - UFMG - Belo Horizonte (MG), Brasil.

(1) Secretaria Estadual de Saúde de Minas Gerais - SESMG - Belo Horizonte (MG), Brasil.

(2) Departamento de Fonoaudiologia, Universidade Federal de Minas Gerais - UFMG - Belo Horizonte (MG), Brasil.

Endereço para correspondência: Stela Maris Aguiar Lemos. Av. Alfredo Balena, 190/069, Santa Efigênia, Belo Horizonte (MG), Brasil, CEP: 30130-

100. E-mail: smarislemos@medicina.ufmg.br

Recebido em: 7/9/2009; Aceito em: 11/11/2010 execução de ações, são consideradas essenciais para a obtenção de melhor qualidade de vida e saúde adequada para a população ${ }^{(1,2)}$. Inserida em muitos sistemas de saúde, a promoção de saúde no Brasil passou a ser um dos principais eixos do Sistema Único de Saúde, trazendo modificações e exigindo que as Instituições de Ensino também sofram alterações, com a finalidade de adequar os cursos à nova realidade do sistema sanitário brasileiro.

O termo "promoção da saúde" é cada vez mais utilizado na área da saúde coletiva/pública, modificando a prática dos profissionais, à medida que amplia o conceito e a visão sobre saúde e que considera o ambiente como um fator importante a ser analisado $^{(1)}$. A ampliação do conceito de saúde fornece suporte às práticas e conceitos de promoção da saúde e é fundamentada na descoberta da insuficiência do modelo biomédico com seu caráter biológico, individualista e direcionado à doença e seus fatores de risco, para a solução dos problemas consequentes da saúde e da doença. As ações de promoção da saúde e suas bases teóricas ultrapassam as ações e níveis de atenção à saúde, não sendo, portanto, anteriores às de prevenção ${ }^{(2,3)}$.

As propostas da promoção da saúde possibilitam aos profissionais comprometidos com a melhoria de vida da população 
uma forma diferenciada de refletir e praticar suas ações em saúde, favorecendo uma visão integral do ser humano ${ }^{(4)}$.

Profissionais da área da Fonoaudiologia inseriram-se nos serviços de saúde pública na década de 80 , refletindo em suas ações conceitos relacionados à recuperação, habilitação e prevenção da saúde, devido à influência do modelo assistencialista vigente e da formação voltada para a atuação em consultórios $^{(5)}$, Porém, a reprodução, em serviços de saúde coletiva, das estratégias utilizadas na prática clínica acarreta gastos importantes para o sistema e não resolve os problemas de saúde da comunidade. Atualmente a análise da intenção de trabalho do fonoaudiólogo recém-formado, demonstra que esta reside no desejo do atendimento em consultórios e na especialização em áreas da Fonoaudiologia ${ }^{(6)}$. Desta forma, as necessidades da política de promoção da saúde ficam à parte, em relação à intenção de trabalho e consequentemente, podem ser negligenciadas na atuação do profissional.

Observa-se que as instituições de ensino formam profissionais cujo perfil não favorece a atuação de acordo com o novo modelo, que exige uma intervenção integral a favor da saúde, por meio de práticas que englobem as propostas de promoção e proteção da saúde, prevenção de doenças, cura e reabilitação ${ }^{(7)}$.

Sabendo-se que a instituição de ensino influencia no processo de formação do profissional, os conceitos adquiridos podem ser condizentes com o tipo de ensino recebido e podem fornecer suporte às futuras ações no ambiente de trabalho. A literatura mostra que o processo de trabalho em saúde é pautado nas ações de ensino e profissionalização ${ }^{(8)}$.

Deste modo, os cursos de graduação devem favorecer, por meio das disciplinas ofertadas, uma visão ampla da saúde, considerando-a como resultado de vários determinantes ${ }^{(7)}$. As modificações do modelo sanitário, assim como as mudanças no sistema de saúde, exigem dos profissionais novas habilidades e competências. Com a finalidade de que os profissionais da saúde incorporem, às suas práticas, estratégias fundamentais de promoção da saúde e de prevenção, programas que visam favorecer uma formação integral e multidisciplinar estão sendo implantados nas instituições de ensino ${ }^{(9)}$. Já existem ações tais como Pró-saúde e Pet-saúde, estruturadas pelos ministérios da saúde e educação, que buscam mudanças na graduação e estão sendo implantadas em diversas universidades brasileiras.

Frente às necessidades do Sistema Único de Saúde e às estratégias utilizadas, é importante estudar a contribuição de cursos de graduação para a formação de profissionais capazes de atuarem em consonância com a realidade e exigências do sistema em vigor.

O presente estudo teve como objetivo caracterizar e comparar o conhecimento dos ingressantes (estudantes do primeiro período) e concluintes (estudantes do oitavo período) de um curso de Fonoaudiologia acerca do tema promoção da saúde.

\section{MÉTODOS}

Trata-se de um estudo descritivo transversal, aprovado pelo Comitê de Ética em Pesquisa da Universidade Federal de Minas Gerais, com parecer $n^{\circ}$ ETIC 279/05. Para cumprir o objetivo foi aplicado um formulário em 92 estudantes do primeiro período (ingressantes) e oitavo período (concluintes) do curso de Fonoaudiologia de uma universidade pública.

$\mathrm{O}$ formulário elaborado foi baseado na leitura de artigos e capítulos de livros que abordavam o tema "promoção da saúde" e composto por questões abertas, fechadas e mistas, que têm como objetivo analisar o conhecimento dos estudantes em Fonoaudiologia sobre o tema. Fez-se necessário realizar um estudo piloto para excluir a possibilidade de existirem questões ambíguas ou de difícil compreensão no formulário. Este foi realizado com oito estudantes do oitavo período do curso de Fonoaudiologia de uma universidade de Belo Horizonte. O formulário foi respondido pelos estudantes, após estarem cientes de que suas respostas não seriam utilizadas na pesquisa. Os estudantes não apresentaram dúvidas com relação às questões do formulário.

Para definição da casuística, foram utilizados os seguintes critérios de inclusão e exclusão:

- foram incluídos os sujeitos que cursavam o primeiro ou oitavo período do curso de Fonoaudiologia que leram e assinaram o Termo de Consentimento Livre e Esclarecido concordando em participar da pesquisa;

- foram excluídos os sujeitos que responderam menos de $80 \%$ das questões do formulário.

Os formulários foram preenchidos pelos estudantes do primeiro e oitavo períodos, no segundo semestre de 2005 e no primeiro semestre de 2006. Desse modo, participaram da pesquisa duas turmas de cada período. Com a finalidade de evitar que os estudantes conhecessem previamente os formulários, estes foram aplicados simultaneamente em cada período.

A Tabela 1 mostra a distribuição dos estudantes que participaram do estudo por período cursado.

Foi realizada a análise descritiva das respostas apresentadas às questões fechadas do formulário, considerando-se a amostra total de estudantes. Foram utilizadas tabelas de frequências para as variáveis categóricas e visualização gráfica por meio de histograma. A comparação entre as respostas do primeiro e oitavo períodos foi realizada por meio de tabelas de asso-

Tabela 1. Participação dos estudantes de Fonoaudiologia

\begin{tabular}{|c|c|c|c|c|c|c|c|c|c|c|}
\hline \multirow{3}{*}{ Estudantes } & \multicolumn{4}{|c|}{2005} & \multicolumn{4}{|c|}{2006} & \multirow{2}{*}{\multicolumn{2}{|c|}{ Total }} \\
\hline & \multicolumn{2}{|c|}{$1^{\circ}$ período } & \multicolumn{2}{|c|}{$8^{\circ}$ período } & \multicolumn{2}{|c|}{$1^{\circ}$ período } & \multicolumn{2}{|c|}{$8^{\circ}$ período } & & \\
\hline & $\mathrm{n}$ & $\%$ & $\mathrm{n}$ & $\%$ & $\mathrm{n}$ & $\%$ & $\mathrm{n}$ & $\%$ & $\mathrm{n}$ & $\%$ \\
\hline Responderam ao formulário & 22 & 88 & 19 & 95,0 & 25 & 100,0 & 26 & 92,8 & 92 & 93,9 \\
\hline Recusaram & 03 & 12,0 & 01 & 5,0 & 00 & 0,0 & 02 & 7,1 & 06 & 6,1 \\
\hline Total matriculado & 25 & 100,0 & 25 & 100,0 & 25 & 100,0 & 28 & 100,0 & 98 & 100,0 \\
\hline
\end{tabular}


ciações, do teste Qui-quadrado e do Teste Exato de Fisher, considerando o nível de significância de $5 \%(0,05)$ em todas as análises.

A análise qualitativa das questões abertas foi realizada utilizando-se a análise de conteúdo tendo como referência cinco categorias de respostas: prevenção; promoção da saúde; prevenção associada à promoção da saúde; reabilitação; não sabe/não foi possível categorizar.

\section{RESULTADOS}

A Tabela 2 apresenta a distribuição da amostra por período e total segundo a vivência de alguma disciplina ou prática curricular/extracurricular que abordou o tema promoção da saúde e conceito de promoção da saúde. A análise evidencia diferenças significativas em todas as situações quando comparados os dois períodos.

A Tabela 3 apresenta respostas às perguntas que requerem conhecimento de conteúdos da política de promoção à saúde segundo o período cursado. Nesta temática a única resposta que evidenciou associação com significância estatística foi a referente à concepção do conceito de saúde como ausência de doença.

Não houve diferenças significativas entre os períodos quanto ao trabalho em promoção da saúde, conforme demonstrado na Tabela 4.

No Quadro 1 está apresentada a categorização das respostas discursivas de ingressantes e concluintes quanto ao conceito de promoção da saúde.

\section{DISCUSSÃO}

Apesar de existirem vários estudos que abordam concepções, conceitos e relatam experiências acerca da promoção de saúde ${ }^{(1,10-17)}$, foram encontrados poucos estudos que discutissem a necessidade do tema ser abordado durante o processo de formação acadêmica ${ }^{(10,18,19)}$.

Os dados revelam que o curso de graduação dos entrevistados propicia o acesso à discussão sobre promoção da saúde, já que os estudantes concluintes apresentaram um número maior de respostas positivas quanto à vivência de disciplinas e práticas na área de promoção da saúde. Esse dado está em consonância com a legislação nacional que preconiza a inclusão em cursos de Instituições de Ensino Superior do conteúdo relacionado ao Sistema Único de Saúde. Salienta-se que a promoção da saúde é um importante eixo deste Sistema (Lei $8.080,1990)$ e das políticas públicas que devem ser garantidas pelo Estado ${ }^{(20)}$.

As respostas evidenciaram um número expressivo de estudantes, mesmo entre os concluintes, que não participaram de atividades extracurriculares, tais como projetos de pesquisa e de extensão que abordaram o tema promoção da saúde. Tal dado pode revelar que grande parte dos estudantes se envolve, ao longo da formação, em atividades de promoção da saúde apenas quando em caráter obrigatório, e que o tema é tratado, sobretudo durante o processo de graduação, sob o ponto de vista teórico. Vale lembrar que as diretrizes curriculares do curso de Fonoaudiologia salientam que o fonoaudiólogo deve ser capaz de atuar em promoção da saúde ${ }^{(21)}$.

Tabela 2. Respostas referentes à vivência do conteúdo Promoção da saúde segundo o período cursado

\begin{tabular}{|c|c|c|c|c|c|c|}
\hline \multirow{3}{*}{$\begin{array}{l}\text { Cursou disciplina do curso de Fonoaudiologia } \\
\text { que discutiu o tema promoção da saúde? }\end{array}$} & \multicolumn{4}{|c|}{ Período do curso } & \multirow{2}{*}{\multicolumn{2}{|c|}{ Total }} \\
\hline & \multicolumn{2}{|c|}{$1^{\circ}$ período } & \multicolumn{2}{|c|}{$8^{\circ}$ período } & & \\
\hline & $\mathrm{n}$ & $\%$ & $\mathrm{n}$ & $\%$ & $\mathrm{n}$ & $\%$ \\
\hline Não & 33 & 70,2 & 00 & 0,0 & 33 & 35,9 \\
\hline Sim & 14 & 29,8 & 45 & 100,0 & 59 & 64,1 \\
\hline Total & 47 & 100,0 & 45 & 100,0 & 92 & 100,0 \\
\hline \multicolumn{7}{|l|}{ Valor de $p=0,000^{*}$} \\
\hline \multirow{3}{*}{$\begin{array}{l}\text { Participou de disciplina prática curricular ou } \\
\text { outra atividade extracurricular que abordou o } \\
\text { tema promoção da saúde? }\end{array}$} & \multicolumn{4}{|c|}{ Período do curso } & \multirow{2}{*}{\multicolumn{2}{|c|}{ Total }} \\
\hline & \multicolumn{2}{|c|}{$1^{\circ}$ período } & \multicolumn{2}{|c|}{$8^{\circ}$ período } & & \\
\hline & $\mathrm{n}$ & $\%$ & $\mathrm{n}$ & $\%$ & $\mathrm{n}$ & $\%$ \\
\hline Não & 44 & 93,6 & 19 & 42,2 & 63 & 68,5 \\
\hline Sim & 03 & 6,4 & 26 & 57,8 & 29 & 31,5 \\
\hline Total & 47 & 100,0 & 45 & 100,0 & 92 & 100,0 \\
\hline \multicolumn{7}{|l|}{ Valor de $p=0,000^{*}$} \\
\hline \multirow{3}{*}{ Sabe o que é promoção de saúde? } & \multicolumn{4}{|c|}{ Período do curso } & \multirow{2}{*}{\multicolumn{2}{|c|}{ Total }} \\
\hline & \multicolumn{2}{|c|}{$1^{\circ}$ período } & \multicolumn{2}{|c|}{$8^{\circ}$ período } & & \\
\hline & $\mathrm{n}$ & $\%$ & $\mathrm{n}$ & $\%$ & $\mathrm{n}$ & $\%$ \\
\hline Não & 22 & 46,8 & 03 & 7,0 & 25 & 27,8 \\
\hline Sim & 25 & 53,2 & 40 & 93,0 & 65 & 72,2 \\
\hline Total & 47 & 100,0 & 43 & 100,0 & 90 & 100,0 \\
\hline Valor de $p=0,000^{*}$ & & & & & & \\
\hline
\end{tabular}

*Valores significativos $(p \leq 0,05)$ - Teste Qui-quadrado 
Tabela 3. Respostas referentes ao conhecimento Promoção da saúde segundo o período cursado

\begin{tabular}{|c|c|c|c|c|c|c|}
\hline \multirow{3}{*}{$\begin{array}{l}\text { Fatores sociais, culturais, econômicos e políticos } \\
\text { podem influenciar no processo saúde-doença? }\end{array}$} & \multicolumn{4}{|c|}{ Período do curso } & \multirow{2}{*}{\multicolumn{2}{|c|}{ Total }} \\
\hline & \multicolumn{2}{|c|}{$1^{\circ}$ período } & \multicolumn{2}{|c|}{$8^{\circ}$ período } & & \\
\hline & $\mathrm{n}$ & $\%$ & $\mathrm{n}$ & $\%$ & $\mathrm{n}$ & $\%$ \\
\hline Não sei & 01 & 2,1 & 00 & 0,0 & 01 & 1,1 \\
\hline Sim & 46 & 97,9 & 43 & 100,0 & 89 & 98,9 \\
\hline Total & 47 & 100,0 & 43 & 100,0 & 90 & 100,0 \\
\hline \multicolumn{7}{|l|}{ Valor de $p=1,000$} \\
\hline \multirow{3}{*}{$\begin{array}{l}\text { Presença de saúde é sinônimo de ausência de } \\
\text { doença? }\end{array}$} & \multicolumn{4}{|c|}{ Período do curso } & \multirow{2}{*}{\multicolumn{2}{|c|}{ Total }} \\
\hline & \multicolumn{2}{|c|}{$1^{\circ}$ período } & \multicolumn{2}{|c|}{$8^{\circ}$ período } & & \\
\hline & $\mathrm{n}$ & $\%$ & $\mathrm{n}$ & $\%$ & $\mathrm{n}$ & $\%$ \\
\hline Não & 35 & 77,8 & 42 & 97,7 & 77 & 87,5 \\
\hline Sim & 02 & 4,4 & 00 & 0,0 & 02 & 2,3 \\
\hline Não sei & 08 & 17,8 & 01 & 2,3 & 09 & 10,2 \\
\hline Total & 45 & 100,0 & 43 & 100,0 & 88 & 100,0 \\
\hline \multicolumn{7}{|l|}{ Valor de $p=0,012^{*}$} \\
\hline \multirow{3}{*}{$\begin{array}{l}\text { Todos os setores da sociedade têm que se } \\
\text { responsabilizar pela melhoria da qualidade de } \\
\text { vida e saúde da comunidade? }\end{array}$} & \multicolumn{4}{|c|}{ Período do curso } & \multirow{2}{*}{\multicolumn{2}{|c|}{ Total }} \\
\hline & & & & & & \\
\hline & $\mathrm{n}$ & $\%$ & $\mathrm{n}$ & $\%$ & $\mathrm{n}$ & $\%$ \\
\hline Não & 01 & 2,1 & 00 & 0,0 & 01 & 1,1 \\
\hline Sim & 44 & 93,6 & 44 & 100,0 & 88 & 96,7 \\
\hline Não sei & 02 & 4,3 & 00 & 0,0 & 02 & 2,2 \\
\hline Total & 47 & 100,0 & 44 & 100,0 & 91 & 100,0 \\
\hline \multicolumn{7}{|l|}{ Valor de $p=0,495$} \\
\hline \multirow{3}{*}{$\begin{array}{l}\text { Desenvolver nos indivíduos a auto-estima e a } \\
\text { responsabilidade é uma forma de se promover } \\
\text { saúde? }\end{array}$} & \multicolumn{4}{|c|}{ Período do curso } & \multirow{2}{*}{\multicolumn{2}{|c|}{ Total }} \\
\hline & \multicolumn{2}{|c|}{$1^{\circ}$ período } & \multicolumn{2}{|c|}{$8^{\circ}$ período } & & \\
\hline & $\mathrm{n}$ & $\%$ & $\mathrm{n}$ & $\%$ & $\mathrm{n}$ & $\%$ \\
\hline Não & 01 & 2,1 & 00 & 0,0 & 01 & 1,1 \\
\hline Sim & 43 & 91,5 & 40 & 93,0 & 83 & 92,2 \\
\hline Não sei & 03 & 6,4 & 03 & 7,0 & 06 & 6,7 \\
\hline Total & 47 & 100,0 & 43 & 100,0 & 90 & 100,0 \\
\hline Valor de $p=1,000$ & & & & & & \\
\hline
\end{tabular}

*Valores significativos $(p \leq 0,05)$ - Teste Exato de Fisher

Os dados evidenciam que mais da metade dos ingressantes $(53,2 \%)$ chega à faculdade com a crença de que sabem o que é promoção de saúde. Neste caso, ressalta-se a importância da universidade no processo de construção e adequação dos conceitos $^{(9)}$, reduzindo-se, assim, a possibilidade dos conceitos empíricos permanecerem presentes ao longo do curso.

As respostas dos estudantes mostram que a necessidade da realização de ações em promoção de saúde é mencionada tanto pelos ingressantes quanto pelos concluintes. Portanto, durante o processo de formação acadêmica, este tema deve ser discutido e abordado. Os resultados do presente estudo corroboram a literatura que afirma a necessidade do fonoaudiólogo reconhecer a importância das ações em promoção da saúde ${ }^{(22)}$. Em relação à formação acadêmica estudos demonstram a necessidade de aproximação das ações dos fonoaudiólogos às propostas do Sistema Único de Saúde, destacando a relevância de uma formação profissional adequada ao modelo de promoção da saúde ${ }^{(16,23)}$. Esses achados corroboram estudos que referem que o fonoaudiólogo deve realizar ações que contribuam para a promoção da saúde e que relatam a necessidade do profissional exercer suas ações em concordância com os princípios do Sistema Único de Saúde ${ }^{(5,23)}$.

Embora a maioria dos estudantes dos dois grupos tenha relatado querer atuar com promoção da saúde, quase $25 \%$ da amostra de concluintes disse que não sabe ou não pretende atuar com promoção da saúde. Diante da procura crescente de concursos públicos, esse dado pode indicar um futuro problema que esses profissionais irão encontrar ao ingressarem no mercado de trabalho, principalmente se forem atuar no Sistema Único de Saúde, que preconiza em sua estruturação e nos objetivos o desenvolvimento de ações e estratégias voltadas a promoção da saúde ${ }^{(20)}$.

Estudos evidenciam a influência de diversos fatores na saúde da população ${ }^{(5,12,13,24,25)}$ e portanto é necessário que, ao ingressar no SUS, o profissional reconheça a necessidade de 
Tabela 4. Respostas referentes às percepções quanto ao trabalho em Promoção da saúde segundo o período cursado

\begin{tabular}{|c|c|c|c|c|c|c|}
\hline \multirow{3}{*}{$\begin{array}{l}\text { Considera importantes ações que } \\
\text { visam à promoção de saúde? }\end{array}$} & \multicolumn{4}{|c|}{ Período do curso } & \multirow{2}{*}{\multicolumn{2}{|c|}{ Total }} \\
\hline & \multicolumn{2}{|c|}{$1^{\circ}$ período } & \multicolumn{2}{|c|}{$8^{\circ}$ período } & & \\
\hline & $\mathrm{n}$ & $\%$ & $\mathrm{n}$ & $\%$ & $\mathrm{n}$ & $\%$ \\
\hline Não se aplica & 01 & 2,2 & 00 & 0,0 & 01 & 1,1 \\
\hline Sim & 45 & 97,8 & 44 & 100,0 & 89 & 98,9 \\
\hline Total & 46 & 100,0 & 44 & 100,0 & 90 & 100,0 \\
\hline \multicolumn{7}{|l|}{ Valor de $p=1,000$} \\
\hline \multirow{3}{*}{ Pretende atuar com promoção da saúde? } & \multicolumn{4}{|c|}{ Período do curso } & \multirow{2}{*}{\multicolumn{2}{|c|}{ Total }} \\
\hline & \multicolumn{2}{|c|}{$1^{\circ}$ período } & \multicolumn{2}{|c|}{$8^{\circ}$ período } & & \\
\hline & $\mathrm{n}$ & $\%$ & $\mathrm{n}$ & $\%$ & $\mathrm{n}$ & $\%$ \\
\hline Não & 00 & 0,0 & 02 & 5,0 & 02 & 2,3 \\
\hline Sim & 34 & 72,3 & 30 & 75,0 & 64 & 73,6 \\
\hline Não sei & 13 & 27,7 & 7 & 17,5 & 20 & 23,0 \\
\hline Não se aplica & 00 & 0,0 & 01 & 2,5 & 01 & 1,1 \\
\hline Total & 47 & 100,0 & 40 & 100,0 & 87 & 100,0 \\
\hline Valor de $p=0,147$ & & & & & & \\
\hline
\end{tabular}

Teste Exato de Fisher $(p \leq 0,05)$

atuar em promoção da saúde e da integração de diferentes setores para obtenção de qualidade de vida adequada ${ }^{(2,3,13,15,26)}$.

Outro dado importante para o profissional é reconhecimento da relevância da promoção da autonomia do indivíduo, capacitando-o a melhorar sua própria qualidade de vida e saúde $^{(2,11,14,21)}$. No presente estudo as perguntas relacionadas a este aspecto não evidenciaram diferenças com significância estatística. Ingressantes e concluintes apresentaram distribuição similar das respostas. Esses achados remetem a estudos que afirmam a necessidade de se proporcionar ao indivíduo a capacidade de melhorar sua própria qualidade de vida e saú$\mathrm{de}^{(2,11,14)}$ e portanto, leva a pensar na necessidade de discutir no espaço acadêmico a produção do cuidado e a promoção da saúde tendo como referência educação em saúde, empoderamento e práticas de autocuidado ${ }^{(26,27)}$.

Os dados do presente estudo revelam que não há relação entre perceber a importância do envolvimento de toda a sociedade na melhoria da qualidade de vida e saber o que é promoção da saúde. Porém, observa-se que estudantes ingressantes e concluintes concordam com a necessidade da participação de diferentes setores para se alcançar uma adequada qualidade de vida, resultados que corroboram estudos que lembram a importância de melhorar a qualidade de vida com as propostas de promoção da saúde ${ }^{(2,15,24,26)}$ e corroboram, ainda, as políticas públicas que estabelecem que a criação de ambientes favoráveis é uma das estratégias da promoção da saúde ${ }^{(11)}$.

Acreditar na influência dos múltiplos determinantes no processo saúde-doença não é um fator que determina saber o que é promoção da saúde e as respostas dos ingressantes e concluintes corroboram vários estudos que lembram a influência múltipla de determinantes na saúde ${ }^{(5,12,13,24,25,27)}$. Vale destacar que, apesar da maioria dos ingressantes e parte dos concluintes ter dúvidas quanto ao conceito de promoção da saúde, apenas um dos sujeitos da pesquisa não reconheceu a influência de determinantes sociais, econômicos e políticos na saúde da população. Assim, quase a totalidade da amostra reconhece a importância de múltiplos determinantes na saúde da população.

Os dados obtidos demonstram a importância da formação acadêmica na futura atuação do profissional. E, para contribuir na atuação profissional pautada nas políticas públicas de saúde e educação, verifica-se que a promoção da saúde deve ser abordada durante o processo de formação ${ }^{(16,24)}$.

A análise das respostas dos ingressantes à pergunta "O que você entende como promoção da saúde?”, demonstra que 25 respostas abordam temas que se referem à promoção da saúde e 19 referem-se a temas de prevenção. Em ambas as categorias nota-se que os temas relatados englobam conceitos empíricos como "evitar doenças", "conscientizar a população" e "vida saudável". Esses achados corroboram estudos que afirmam que existe uma confusão entre os termos prevenção e promoção da saúde ${ }^{(28,29)}$.

A análise da questão aberta foi fundamental para a compreensão dos conceitos utilizados pelos estudantes, pois apesar de $93 \%$ dos concluintes relatarem que sabem o que é promoção da saúde, verificamos que 11 respostas abordam temas apenas de prevenção ou de prevenção e promoção da saúde. Portanto, é possível afirmar que, apesar do processo de formação favorecer a aquisição de conceitos adequados de promoção da saúde, permanece a confusão entre este termo e o de prevenção de doenças.

Estudos mostram que Fonoaudiólogo reflete, na prática, a formação que obteve na instituição de ensino, reproduzindo, na saúde coletiva, conceitos basicamente assistencialistas, e há dificuldade de adequação da prática fonoaudiológica ao modelo de promoção da saúde, devido à formação clínica. Muitas vezes as instituições de ensino priorizam uma formação que não favorece ações condizentes com o modelo desejável de atuação em saúde e deste modo, desenvolvimento e avaliação de projetos de promoção da saúde são estratégias pouco utilizadas no processo de ensino-aprendizagem em cursos de Fonoaudiologia $^{(6,28,29)}$. 
Quadro 1. Respostas dos estudantes ingressantes e concluintes à pergunta "O que você entende como promoção da saúde?"

\begin{tabular}{|c|c|c|c|c|}
\hline & Ingressantes & & Concluintes & \\
\hline Categorias & $\begin{array}{l}\text { Conteúdos/conceitos } \\
\text { respostas apresentadas }\end{array}$ & $\mathrm{n}$ & $\begin{array}{l}\text { Conteúdos/conceitos } \\
\text { respostas apresentadas }\end{array}$ & $\mathrm{n}$ \\
\hline Prevenção & $\begin{array}{l}\text { prevenir/evitar doenças; } \\
\text { orientar; } \\
\text { cuidar; } \\
\text { conscientizar a população sobre doenças; } \\
\text { discutir higiene. }\end{array}$ & 19 & $\begin{array}{l}\text { evitar doenças; } \\
\text { palestras; } \\
\text { folhetos informativos; } \\
\text { eliminar fatores de risco. }\end{array}$ & 02 \\
\hline Promoção da saúde & $\begin{array}{l}\text { trabalho interdisciplinar/ multidisciplinar; } \\
\text { qualidade de vida; } \\
\text { vida saudável; } \\
\text { bem estar social, mental e fisiológico. }\end{array}$ & 25 & $\begin{array}{l}\text { empoderamento; } \\
\text { qualidade de vida; } \\
\text { bem estar biopsicossocial; } \\
\text { integralidade; } \\
\text { co-responsabilização; } \\
\text { criar ambientes favoráveis; } \\
\text { interdisciplinaridade/ multidisciplinaridade; } \\
\text { ações em nível individual e coletivo. }\end{array}$ & 33 \\
\hline $\begin{array}{l}\text { Prevenção e } \\
\text { promoção da saúde }\end{array}$ & $\begin{array}{l}\text { prevenção de patologias associada à } \\
\text { melhoria da qualidade de vida. }\end{array}$ & 01 & $\begin{array}{l}\text { evitar doenças; } \\
\text { conscientizar/informar; } \\
\text { detecção precoce; } \\
\text { ações de atenção primária, secundária e } \\
\text { terciária; } \\
\text { bem estar biopsicossocial; } \\
\text { qualidade de vida; } \\
\text { interdisciplinaridade; } \\
\text { co-responsabilização; }\end{array}$ & 09 \\
\hline Recuperação & $\begin{array}{l}\text { rede hospitalar; } \\
\text { assistência }\end{array}$ & 05 & habilitar, reabilitar, aperfeiçoar. & 02 \\
\hline $\begin{array}{l}\text { Não sabe/não foi } \\
\text { possível categorizar }\end{array}$ & & 05 & & 01 \\
\hline
\end{tabular}

Nota-se, ainda, que há a necessidade de modificar a formação dos profissionais, para que possam atuar em consonância com o sistema de saúde, incorporando, em suas práticas, ações de promoção da saúde ${ }^{(10)}$. Contudo já se observa a inclusão da prática voltada para a promoção da saúde em cursos de Fonoaudiologia, como demonstra a literatura ${ }^{(30)}$, e também a inserção de estudantes de Fonoaudiologia em projetos de extensão que visam a promoção da saúde ${ }^{(29)}$.

Observa-se um reduzido número de trabalhos que pesquisam sobre a temática da promoção da saúde durante a formação profissional. Assim, verifica-se a necessidade da continuidade da realização de estudos na área, sobretudo aqueles voltados às temáticas de aquisição de conceitos e desenvolvimento de habilidades e competências para atuação em promoção da saúde. Torna-se necessário, também, avaliar como a implementação das Diretrizes Curriculares Nacionais em Fonoaudiologia e o desenvolvimento das ações do Pró-saúde e PET-saúde produziram mudanças na formação e na prática profissional do fonoaudiólogo.

\section{CONCLUSÃO}

Diante dos resultados encontrados pode-se concluir que a graduação em Fonoaudiologia contribui para a construção do conceito de promoção da saúde, pois há diferença conceitual entre ingressantes e concluintes. Contudo existe uma confusão entre os termos prevenção e promoção da saúde, tanto entre ingressantes quanto entre concluintes, apesar de tal confusão ocorrer em menor quantidade entre os concluintes. 


\begin{abstract}
Purpose: To characterize and compare the knowledge of freshmen (1st period) and senior (8th period) undergraduate Speech-Language Pathology and Audiology students regarding health promotion. Methods: Cross-sectional study that involved the application of 92 forms to 1st and 8th period Speech-Language Pathology and Audiology students. Data analysis was conducted in three stages: descriptive analysis of closed questions, statistical analysis crossing the answers of both groups, and qualitative analysis of the open questions of the form. Results: Most interviewees attended classes directed to the theme of health promotion; more than a third of them participated in extracurricular activities or practices on the theme. Most subjects reported to know the concept of health promotion and to intend to create health promotion actions. Freshmen students reported empirical concepts of health promotion, and confused prevention with promotion. Among senior students, the confusion exists in smaller quantities. Thus, although the training process favor the acquisition of adequate concepts of health promotion, terminology confusion still remains. Conclusion: The undergraduate program in Speech-Language Pathology and Audiology of the students interviewed contributes for the construction of the concept of health promotion, however, there is confusion between the terms health prevention and promotion among both groups of students. Data evidence the importance to discuss the health promotion theme during the formation process of speech-language pathologists and audiologists.
\end{abstract}

Keywords: Speech, language and hearing sciences/education; Health promotion; Health knowledge, attitudes, practice; Health education; Education, higher; Questionnaires

\title{
REFERÊNCIAS
}

1. Czeresnia D. O conceito de saúde e a diferença entre prevenção e promoção. In: Czeresnia D, Freitas CM. Promoção da Saúde: conceitos, reflexões e tendências. Rio de Janeiro: Fiocruz; 2003. p.39-54.

2. Marcondes WB. Convergência de referências na promoção da saúde. Saúde Soc. 2004;13(1):5-13.

3. Moysés SJ, Moysés ST, Krempel MC. Avaliando processo de construção de políticas públicas de promoção de saúde: a experiência de Curitiba. Ciênc Saúde Coletiva. 2004;9(1):627-41.

4. Bydlowski CR, Westphal MF, Pereira IM. Promoçäo da saúde: porque sim e porque ainda não!. Saúde Soc. 2004;13(1):14-24.

5. Chun RY. Promoção da saúde e as práticas em Fonoaudiologia. In: Ferreira LP, Befi-Lopes DM, Limongi SC. Tratado de Fonoaudiologia. São Paulo: Roca; 2004. p. 538-43.

6. Silva DG, Sampaio TM, Bianchini EM. Percepções do fonoaudiólogo recém-formado quanto a sua formação, intenção profissional e atualização de conhecimentos Rev Soc Bras Fonoaudiol. 2010;15(1):47-53.

7. Gil CR. Formação de recursos humanos em saúde da família: paradoxos e perspectivas. Cad Saúde Pública. 2005;21(2):490-8.

8. Ceccim RB. Estado, sociedade e formação profissional em saúde: contradições e desafios em 20 anos de sus. Cad Saúde Pública. 2009;25(10):2299-300.

9. Lefevre AM, Cornetta VK. Recursos humanos para a promoção da saúde. In: Lefevre F, Lefevre AM. Promoção de Saúde: a negação da negação. Rio de Janeiro: Vieira e Lent; 2004. p. 11-66.

10. Yen E, Rocha CM, Godue C. Reorientando práticas: educação em saúde através da mobilização de atores, recursos e competências. Olho Mágico. 2005;12(1):267-8.

11. Hespanhol AP, Couto L, Martins C. A medicina preventiva. Rev Port Clin Geral. 2008;24:49-64.

12. Buss PM. Promoção da Saúde e Qualidade de Vida. Ciênc Saúde Coletiva. 2000;5(1):163-77.

13. Lefevre F, Lefevre AM. Promoção de Saúde: a negação da negação. Rio de Janeiro: Vieira e Lent; 2004. A crítica dos conceitos; p.133-46.

14. Campos GW, Barros RB, Castro AM. Avaliação de política nacional de promoção da saúde. Ciênc Saúde Coletiva. 2004;9(3):745-9.

15. Stotz EN, Araújo JW. Promoção da saúde e cultura política: a reconstrução do consenso. Saúde Soc. 2004;13(2): 5-19.

16. Penteado RZ, Servilha EA. Fonoaudiologia em saúde pública/coletiva: compreendendo prevenção e o paradigma da promoção da saúde. Distúrb Comun. 2004;16(1):107-16.

17. Santos LM, Da Ros MA, Crepaldi MA, Ramos LR. Grupos de promoção à saúde no desenvolvimento da autonomia, condições de vida e saúde. Rev Saúde Pública. 2006;40(2):346-52.
18. Chiesa AM, Nascimento DD, Braccialli LA, Oliveira MA, Ciampone MH. A formação de profissionais da saúde: aprendizagem significativa à luz da promoção da saúde. Cogitare Enferm. 2007;12(2):236-40.

19. Machado MF, Monteiro EM, Queiroz DT, Vieira NF, Barroso MG. Integralidade, formação de saúde, educação em saúde e as propostas do SUS: uma revisão conceitual. Ciênc Saúde Coletiva. 2007;12(2):335-42.

20. Brasil. Lei 8.080 de 19 de setembro de 1990. Dispõe sobre as condições para a promoção, proteção e recuperação da saúde, a organização e o funcionamento dos serviços correspondentes e dá outras providências [Internet]. Diário Oficial da República Federativa do Brasil, Brasília (DF); 1990 Set 19 [citado 2006 Jun 9]. Disponível em: http://conselho.saude.gov. br/legislacao/lei8080_190990.htm.

21. Brasil. Ministério da Educação. Parecer n. 1210, de 12 de setembro de 2001. Diretrizes curriculares nacionais dos cursos de graduação em fisioterapia, Fonoaudiologia e terapia ocupacional [Internet]. Diário Oficial da República Federativa do Brasil, Brasília (DF); 2001 Dez 10; Seção 1:22. [citado 2006 Jun 09]. Disponível em: http://portal.mec.gov.br/cne/arquivos/pdf/ pces1210_01.pdf.

22. Arce VA, Lemos SM, Magalhães EM. Promoção da saúde em Fonoaudiologia: relato de experiência em creche. Olho Mágico. 2005;12(1):120

23. Cotta RM, Azeredo CM, Maia TM, Pereira RJ, Marques ES, Schott M, et al. Formação de recursos humanos no SUS: uma realidade ainda pendente. Londrina: Olho Mágico. 2005;12(1):66.

24. Delgado SE. A Fonoaudiologia no contexto da promoção da saúde coletiva materno-infantil. Fono Atual. 2004;29(2):72-5.

25. Brasil, Ministério da Saúde. Comunicação e educação em saúde. O SUS de A a Z: garantindo saúde nos municípios. Brasília (DF): Ministério da Saúde; 2005.

26. Buss PM. Uma introdução ao conceito de promoção da saúde. In: Czeresnia D, Freitas CM. Promoção da Saúde: conceitos, reflexões e tendências. Rio de Janeiro: Fiocruz; 2003. p.15-38.

27. Fleury-Teixeira P, Vaz FA, Campos FC, Álvares J, Aguiar RA, Oliveira VA. Autonomia como categoria central no conceito de promoção de saúde. Ciênc Saúde Coletiva. 2008:13(Supl 2):2115-22.

28. Ros MA. Concepções de saúde e promoção à saúde dos estudantes de Medicina da UFSC. Olho Mágico. 2005;12(1):97-8.

29. Gonçalves CG. Reflexões sobre o percurso da Fonoaudiologia na constituição de um fazer preventivo. Saúde Rev. 2001;3(5/6):55-66.

30. Lemos SM, Furuie RA, Lapa MC, Pereira LD, Hayama ET. Saúde do idoso na atenção primária à saúde: proposta de atuação interdisciplinar. In: 20 Congresso Brasileiro de Extensão Universitária; 2004; Belo Horizonte. 